\title{
MicroRNAs as Modulators of Tumor Metabolism, Microenvironment, and Immune Response in Hepatocellular Carcinoma
}

\author{
Laura Gramantieri ${ }^{\prime}$ \\ Catia Giovannini ${ }^{2,3}$ \\ Fabio Piscaglia (D) 1,4 \\ Francesca Fornari iD 3,5 \\ 'Division of Internal Medicine, \\ Hepatobiliary and Immunoallergic \\ Diseases, IRCCS Azienda Ospedaliero- \\ Universitaria di Bologna, Bologna, Italy; \\ ${ }^{2}$ Department of Experimental, Diagnostic \\ and Specialty Medicine (DIMES), \\ University of Bologna, Bologna, Italy; \\ ${ }^{3}$ Centre for Applied Biomedical Research \\ - CRBA, University of Bologna, St. Orsola \\ Hospital, Bologna, Italy; ${ }^{4}$ Department of \\ Medical and Surgical Sciences, University \\ of Bologna, Bologna, Italy; ${ }^{5}$ Department \\ for Life Quality Studies (QuVi), \\ University of Bologna, Rimini, Italy
}

\begin{abstract}
Hepatocellular carcinoma (HCC) is the second leading cause of cancer-related mortality. Molecular heterogeneity and absence of biomarkers helping patient allocation to the best therapeutic option contribute to poor prognosis in advanced stages. MicroRNAs' (miRNAs) deregulated expression contributes to tumor development and progression and influences drug resistance in HCC. Accordingly, miRNAs have been extensively investigated as both biomarkers and therapeutic targets. The diagnostic and prognostic roles of circulating miRNAs have been ascertained, though with some inconsistencies across studies. From a therapeutic perspective, miRNA-based approaches demonstrated safety profiles and antitumor efficacy in HCC animal models. Nevertheless, caution should be used when transferring preclinical findings to the clinic, due to possible molecular inconsistency between animal models and the heterogeneous patterns of human diseases. A wealth of information is offered by preclinical studies exploring the mechanisms driving miRNAs' aberrant expression, the molecular cascades triggered by miRNAs and the corresponding phenotypic changes. Ex-vivo analyses confirmed these results, further shedding light on the intricacy of the human disease often overcoming pre-clinical models. This complexity seems to be ascribed to the intrinsic heterogeneity of HCC, to different risk factors driving its development, as well as to changes across stages and previous treatments. Preliminary findings suggest that miRNAs associated with specific risk factors might be more informative in defined patients' subgroups. The first issue to be considered when trying to envisage a possible translational perspective is the molecular context that often drives different miRNA functions, as clearly evidenced by "dual" miRNAs. Concerning the possible roles of miRNAs as biomarkers and therapeutic targets, we will focus on miRNAs' involvement in metabolic pathways and in the modulation of tumor microenvironment, to support their exploitation in defined contexts.
\end{abstract}

Keywords: HCC, microRNA, biomarkers

\section{Plain Language Summary}

The prognosis of hepatocellular carcinoma (HCC) in the advanced stages remains poor, even though novel treatments have entered clinical practice in the recent years. Unfortunately, there are no biomarkers of individual prognosis and response to therapies to help patient allocation to treatments. MicroRNAs' deregulated expression contributes to HCC development, progression, and response to treatments, thus this class of molecules represents a possible source of prognostic and predictive biomarkers as well as therapeutic targets to be investigated. Here we focused on some characteristics of microRNAs, which should always be considered, in order to adequately utilize this class of molecules. First, the molecular context dictates relevance and functions of microRNAs and the presence of defined genetic lesions may strongly influence the function of microRNAs. Second, microRNAs participate in metabolic changes, which are a pivotal aspect of
Correspondence: Laura Gramantieri; Francesca Fornari

University of Bologna, Via Massarenti, 9, Bologna, 40138, Italy

Tel/Fax +3905I 2143902

Email laura.gramantieri@aosp.bo.it;

francesca.fornari2@unibo.it 
HCC aggressiveness. Findings collected so far suggest that specific microRNA panels might be informative in different etiologies of background liver disease, as well as in the setting of specific treatments, such as the immune-modulating ones.

\section{Introduction}

Hepatocellular carcinoma (HCC) arises in the background of cirrhosis in about $80-90 \%$ of cases. Main risk factors driving cirrhosis are hepatitis $\mathrm{B}$ and $\mathrm{C}$ virus (HBV and HCV) infection, alcohol intake, aflatoxin exposure, metabolic syndrome and NAFLD, these last ones gaining more and more relevance due to changes in life style and advancements in the cure of hepatitis viruses. ${ }^{1}$ Events leading to HCC development recapitulate the hallmarks of cancer, including increased proliferation, cell cycle alterations, metabolic changes, gaining of invasive and metastatic capabilities, epithelial to mesenchymal transition and stem-like features. The activation of several signaling pathways is associated with all these alterations, which are usually combined. Remarkably, the corresponding phenotypic changes were incorporated into molecular classifications of $\mathrm{HCC}$ as criteria for subgroups' identification. ${ }^{2}$ Despite the fact that a molecular classification of HCC has been proposed and validated, this tool never entered clinical practice. Indeed, the imaging-driven diagnostic work-up of HCC, instead of tissue sampling, has contributed to hampering the introduction and the development of molecular biomarkers in clinical practice.

MicroRNAs (miRNAs) are a class of small RNAs able to modulate mRNA expression either by impairing translation, or by promoting its degradation. Mechanistically, they bind to target mRNAs by base pair complementarity between their seed sequence and the matching sequence in the $3^{\prime}$ untranslated region (3'UTR) of the target mRNA. Each miRNA recognizes complementary sequences in hundreds of mRNAs and, at the same time, each mRNA can be targeted by many miRNAs, with variable base-pairing strength of the miRNA-target duplex. This scenario becomes more and more complex with the recognition of feedback and feedforward loops underlying the physiologic role of miRNAs in the fine-tuning of mRNA expression at multiple levels. The disruption of physiologic conditions occurring in cancer cells alters the relationships between miRNAs and targets, as frequently reported. In specific contexts, some miRNA functions become prevalent, gaining relevance in the development of cancer hallmarks, as shown by animal models and ex-vivo series.
Studies have shown that tissue miRNA signatures are associated with HCC stage, viral etiology, aggressive phenotype, and molecular subgroups. ${ }^{3}$ During liver carcinogenesis, miRNAs were shown to contribute to almost all features of the neoplastic cells, contributing to increased proliferation, survival and migration, as well as to reduced apoptosis and altered drug sensitivity. Beside mechanistic insights, they were also proposed as putative biomarkers of diagnosis, prognosis, and response to treatments. Indeed, miRNA-based signatures define prognostic subgroups of HCC and their circulating fraction holds promise as a non-invasive tool to predict response to systemic therapies. The relevant molecular heterogeneity of HCC and the use of different experimental approaches and control groups in profiling studies have limited the validation of inter-laboratory findings. Despite the discrepancies across different studies, several miRNAs are consistently deregulated in HCC tissue, such as miR-122, let-7, miR-21, miR-221-222, miR-30 family, miR-26, miR29a/b/c, miR-200a/b/c, miR-199a, miR-34a, and C19 miRNA cluster (C19MC), highlighting their contribution to hepatocyte de-differentiation and tumor progression. A huge amount of data allows us to hypothesize regarding their role as biomarkers and possible novel therapeutic targets.

Hereafter we will focus on a few examples explaining the role of the molecular context in dictating miRNA specific behavior. Next, we will review miRNAs' involvement in cellular metabolism and mechanisms occurring in metabolic-related liver diseases, aiming at identifying possible novel biomarkers and therapeutic candidates to be proposed in this rapidly spreading condition. Then we will focus on the role of miRNAs in the crosstalk between microenvironment cell populations and in the modulation of the immune checkpoint molecules, due to the emerging perspective of immuno-oncology in HCC.

\section{Molecular Context and "Dual" miRNAs}

With the deepening of the knowledge on miRNA functions, the role of the molecular context has gained strong relevance. The original paradigm linking one miRNA to a single target has become more complex. Indeed, it is not surprising that both oncogenes and tumor suppressor genes have been identified among putative targets of the same miRNA. Of course, most of the validated targets are those that confirm the working hypothesis, thus most mechanistic studies provide focused evidence, disregarding a comprehensive evaluation. By questioning 
bioinformatics tools, it appears evident that one miRNA might target both positive and negative regulators of the same molecular pathway. From a physiologic perspective, this can be ascribed to the role of miRNAs as fine regulators of signaling pathways. However, in the neoplastic context, where the constitutive expression of molecules belonging to a specific signaling cascade is altered and different from the physiologic counterpart, it is difficult to affirm, "a priori", the net result of miRNA deregulation, especially when the expression of targets is unknown. As an example we can cite miR-519d that belongs to the C19MC and is aberrantly overexpressed, with a mean 10fold increase, in half of HCCs. MiR-519d promotes cell proliferation, invasion but also drug sensitization of HCC cells through CDKN1A/p21, AKT3 and PTEN targeting. ${ }^{4}$ Notably, miR-519d targets both an oncogene (AKT3) and a tumor suppressor gene (PTEN), belonging to the same signaling cascade. On one side, this explains its dual role in other cancer types, ${ }^{5}$ on the other side it is intuitive that constitutive expression of AKT3 and PTEN is crucial to figure out the net functional relevance of miR-519d overexpression. Similarly, miR-221 effects were found to be partly dependent on the molecular context. MiR-221 promotes proliferation by inhibiting cell cycle checkpoints such as p27 and p57 and undermines apoptosis by targeting anti-apoptotic factors such as Bmf and Puma. However, the presence of wild type (WT) TP53 enables the establishment of a miR-221/mdm2/p53 loop that is responsible for the concomitant activation of $\mathrm{p} 53$ dependent pathways triggering apoptosis and sensitization to genotoxic damage. Conversely, in TP53-mutated contexts, the miR-221/mdm2/p53 loop cannot work, and miR221 expression exerts pro-cancerous behavior only. In principle, these findings outline TP53 status as a promising condition to be evaluated when therapeutic inhibition of miR-221 is considered. Accordingly, downregulated miR-30e-3p is associated with poor prognosis, proliferation, stemness and drug resistance in $\mathrm{HCC}$, and simultaneously targets oncogenes (MDM2, EpCAM) and tumor suppressor genes $(C D K N 1 B / \mathrm{p} 27$ and $P T E N)$. We recently reported its "dual" role in $\mathrm{HCC}$, highlighting opposite effects based on different molecular backgrounds and TP53 mutations. Indeed, in TP53 WT HepG2 cells, miR-30e-3p behaves as a tumor suppressor miRNA due to mdm2 targeting, leading to p53 pathway activation. Conversely, in the absence of a functional TP53, mdm2 silencing becomes irrelevant and p27 and PTEN inhibition prevails, leading to increased proliferation, stemness properties, and drug resistance. ${ }^{6}$ The relevance of TP53 as a modulator of miRNA functions is not a marginal observation (Figure 1), since in the case of HCC, mutated isoforms of TP53 can be recognized in approximately $30 \%$ of cases and its impaired function is recognizable in an even wider proportion of cases. These examples highlight the importance of accurate molecular characterization when defining miRNA functions in cancer, and especially when therapeutic exploitation might be envisaged.

\section{MicroRNAs and Metabolism in HCC}

Metabolic reprogramming is a survival strategy of cancer cells to adapt to harsh environments where glucose and oxygen supplies are limited. Glucose uptake is increased in cancer cells which shift their metabolism to "aerobic glycolysis" leading to energy production independent from oxygen availability, which can be insufficient especially in fast-growing tumors. Metabolic reprogramming, also known as Warburg effect, is considered a hallmark of cancer and is associated with poor prognosis. ${ }^{7}$ Cancer cells adopt this metabolic change to face increased energetic and anabolic requirements needed for rapid proliferation and clonal expansion. The Warburg effect favors the employment of glucose as a carbon source to obtain energy and metabolic intermediates from non-oxidative phosphorylation giving advantages to highly proliferating malignant cells. ${ }^{8}$ In addition to the Warburg effect, the stabilization of hypoxia-inducible factor 1 (HIF-1 $\alpha$ ) enables the reduction of mitochondria-associated reactive oxygen species (ROS) that have deleterious effects on highly proliferating cells. ${ }^{9}$

The Warburg effect is a very early event in hepatocarcinogenesis. Indeed, preneoplastic foci adopt a metabolic shift to progress toward full-blown HCCs with decreased oxygen consumption, inhibition of oxidative phosphorylation (OXPHOS) and redirection of glucose utilization toward the phosphate pentose pathway (PPP). All these metabolic alterations are governed by transcriptional changes directed by NRF2-mediated increase of the PPPlimiting enzyme glucose 6-phosphate dehydrogenase (G6PD), and by the increased expression of the mitochondrion chaperone TRAP1 that inhibits respiratory complex IV and succinate dehydrogenase (SDH). MiR-1 expression is regulated by NRF2 and takes part in rewiring these metabolic circuitries by targeting G6PD in preclinical models. ${ }^{10}$ These findings suggest that miR-1 upregulation might be investigated as a biomarker of HCC sustained by a glycolytic phenotype. Interestingly, this field of 


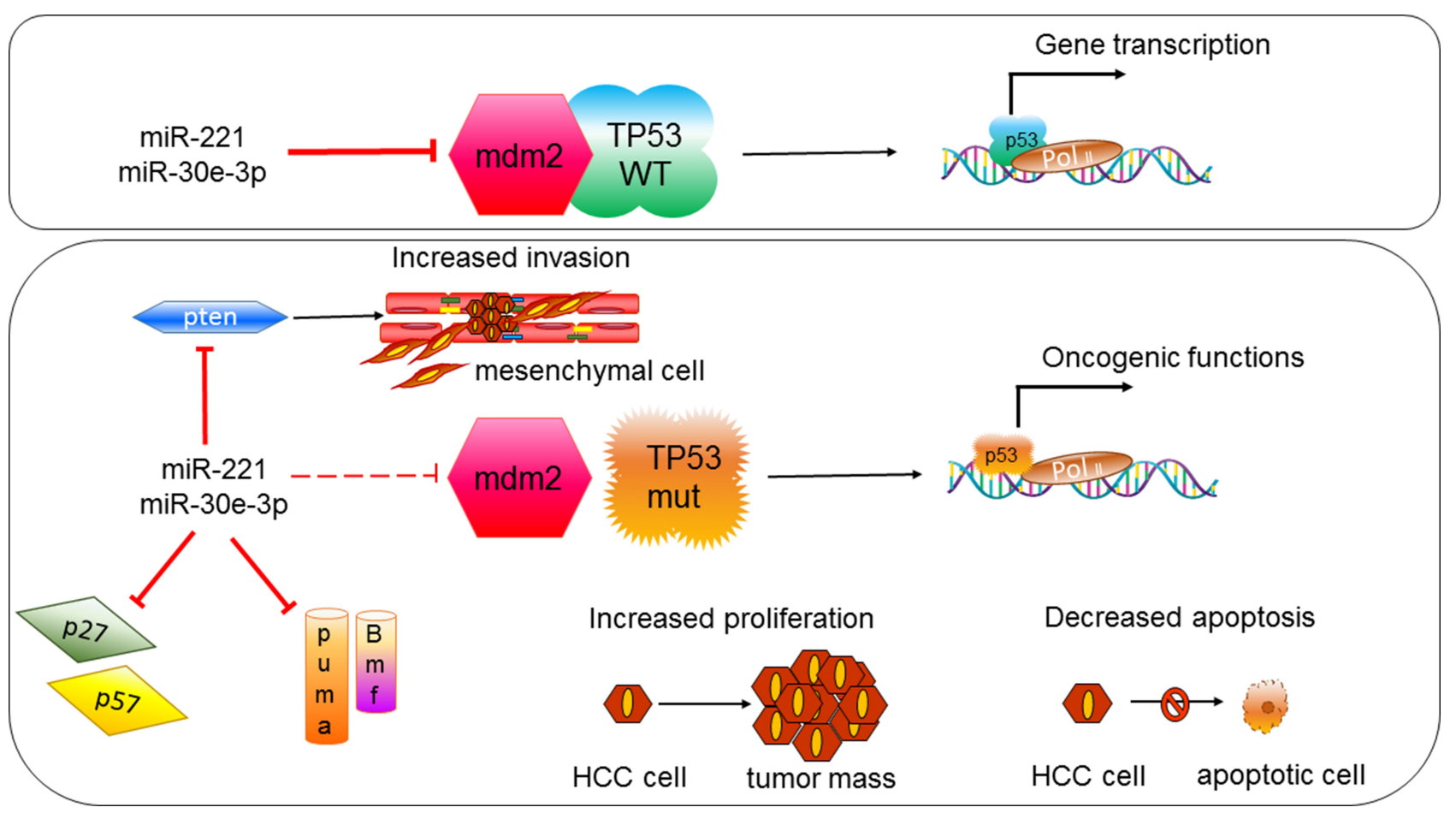

Figure I TP53 status and "dual" microRNAs in HCC.

Notes: Upper panel: in the presence of wild type (WT) TP53, mdm2 targeting by miR-22I and miR-30e-3p activates the p53 axis. Lower panel: in the presence of mutant (mut) TP53, mdm2 targeting by miR-22I and miR-30e-3p is ineffective favoring the regulation of tumor suppressor genes leading to increased cell proliferation (by blocking p27 and p57), invasion (by blocking PTEN), and reduced apoptotic cell death (by blocking puma and bmf).

investigation is gaining more and more relevance, also in light of advisable therapeutic implications. ${ }^{11-13}$

Wang et al recently underscored the relevance of metabolic changes as drivers and prognostic factors in HCC. ${ }^{14}$ These authors analyzed the ICGC and the TCGA data sets and found 178 commonly deregulated metabolic genes. Among these, 17 metabolism-related genes displayed an association with overall survival in HCC patients and a signature of six genes was further validated in the TCGA data set as an independent prognostic tool. This study remarks on the role of deranged metabolism as a driver of HCC aggressiveness, with prognostic and predictive implications. In the context of oncometabolic pathways, miR-21 was recently reported to play a pivotal role in the progression from NASH, to NAFLD to NAFLDrelated HCC. ${ }^{15}$ MiR-21 directly participates in lipid metabolism disorders, inflammation, fibrosis and oncogenic changes by modulating multiple signaling pathways, among which PI3K/AKT, TGF-beta, and STAT3 together with several oncogenic factors. The contribution of miR21 to NASH, NAFLD, and NAFLD-related HCC development was demonstrated both in a zebrafish HCC model and in human HCC. Thus, miR-21 resumes the multitarget function of miRNAs which are able to impact on multiple and different changes, promoting the development of diseases and cancer.

MiRNA signatures differentiate $\mathrm{HCV}$ and $\mathrm{HBV}$ infected livers. Conversely, very few data are available on miRNA deregulation in metabolic liver diseases and HCC. The hepatospecific miR-122 is deregulated early in liver cancer progression. Its role in lipid metabolism and cholesterol synthesis is well known and has been studied from a therapeutic perspective with the in vivo delivery of anti-sense oligonucleotides ${ }^{16}$ and in miR-122 knockout (KO) mouse models. ${ }^{17}$ Among miR-122 direct targets, human Agpat1 and Dgat1 mRNAs, involved in triglyceride synthesis, were validated in the liver where a feedback loop with free fatty acids is established. ${ }^{18}$ Esau et al inhibited miR-122 by $90 \%$ in liver tissue. They employed a diet-induced obesity mouse model using antisense oligonucleotides, to demonstrate a reduction in plasma cholesterol, resulting from a decrease in hepatic fatty-acid and cholesterol synthesis, and an increase of hepatic fatty-acid oxidation, ultimately leading to a significant improvement in liver steatosis. Over a 4 week period of $90 \%$ inhibition of the most abundant liver miRNA, any untoward effect was not observed. The demonstration of miR-122 as a key regulator of cholesterol and fatty acid (FA) metabolism in 
the adult liver makes this miRNA an attractive putative therapeutic target for metabolic disease unresponsive to style life corrections and traditional drugs. Since miR122 is essential for $\mathrm{HCV}$ replication, its inhibition was exploited in human trials to prevent $\mathrm{HCV}$ replication in the pre-direct antiviral agents (DAAs) era. ${ }^{19}$ Initial results were favorable in terms of safety and prolonged dosedependent reduction in HCV RNA levels without evidence of viral resistance in patients with chronic HCV genotype 1 infection (ClinicalTrials.gov number, NCT01200420). However, the high effectiveness of DAAs prevented future development of this approach toward HCV infection. Meanwhile, the "metabolic epidemic" seems to acquire more and more relevance in the scenario of chronic liver diseases and HCC, now making the miR-122 manipulation approach interesting from a "metabolic" rather than a "viral" perspective.

MiR-122 deregulation is observed not only in cancer tissue but also in precancerous lesions such as liver cirrhosis and viral hepatitis where it is associated with impairment of mitochondrial function, showing a positive correlation with mitochondrion-associated genes (secondary targets) involved in amino acids and fatty acids metabolism. In agreement, miR-122 secondary targets are expressed to a lesser extent in HCC and non-tumor tissue from HCC patients with poorer prognosis, highlighting their central role in tumor aggressiveness. ${ }^{20}$ An inverse correlation between miR-122 and pyruvate kinase $(\mathrm{PK})$ mRNA was detected in HCC and functional studies revealed PKM2, the most abundant pyruvate kinase isoenzyme in liver tumors, as a direct target of miR-122 in HCC cells. PK is a glycolytic enzyme involved in the last step of glycolysis responsible for the de-phosphorylation of phosphoenolpyruvate (PEP) to pyruvate. MiR-122 overexpression in HCC cells decreases lactate production and increases oxygen consumption, reversing their oxygenindependent glycolytic metabolism. PK expression is associated with overall survival in HCC patients, suggesting the deep implication of an miRNA-based therapeutic intervention in cancer metabolic pathways. ${ }^{21}$ Indeed, considering the high avidity of hepatocytes to small RNAs uptake, the direct injection of miR-122 into the tumor nodule might be proposed during standard of care procedures such as trans-arterial chemo-embolization (TACE), thus allowing the delivery of miRNAs analogs quite entirely into the tumor nodule, thus avoiding distant unwanted side effects. We should also consider that other targets of miR-122 such as cyclin G1, ADAM10,
ADAM17 and IGF1R might be inhibited as well, increasing the anti-oncogenic effect of miR-122 restoration. Remarkably, other signaling pathways converge on the modulation of PK. Among these, the mineralocorticoid receptor (MR) is downregulated in $80 \%$ of HCCs due to chromosome deletion or histone deacetylation and its low expression is associated with increased lactate production, increased proliferation and tumorigenic properties as well as worse survival of HCC patients. Specifically, MR transcriptionally activates miR-338-3p which, in turn, inhibits liver and red blood cells pyruvate kinase isoforms (PKLR), interfering with the Warburg effect in HCC cells. $^{22}$ Circular RNA MAT2B (circMAT2B) is upregulated in $\mathrm{HCC}$ where it is associated with poor prognosis and altered glucose metabolism, as detected by positron emission tomography combined with computer tomography (PET/CT) imaging with $\left[{ }^{18} \mathrm{~F}\right]$-Fluorodeoxyglucose ( $\left.\left[{ }^{18} \mathrm{~F}\right]-\mathrm{FDG}\right)$. CircMAT2B upregulation contributes to the metabolic shift of HCC cells by sequestering miR-338-3p due to its sponging activity, disabling the regulation of its target gene PKM2 leading to increased proliferation, invasion, spheroid formation, and organoid dimensions especially in hypoxic conditions. $^{23}$ Thus, the combined assay of miR-1, miR-122 and miR-338-3p might be investigated as a possible surrogate biomarker of the Warburg phenotype in HCC.

Gluconeogenesis is at the base of glucose homeostasis and it is altered in liver cancer too, leading to enhanced glycolysis. Mice fed a choline-deficient diet (CDD) develop $\mathrm{HCC}$ in a non-alcoholic steatohepatitis (NASH) background and show marked reduction of the transcription factor PPARGC1A $(P g c-1 a)$ that regulates the expression of key gluconeogenic enzymes such as glucose-6-phosphatase $(G 6 p c)$, phosphoenolpyruvate carboxykinase (Pepck) and fructose-1,6-phosphatase (Fbpl). CDD-fed mice display reduced serum glucose levels and, on the contrary, increased interleukin-6 (IL-6) levels activating the STAT3 pathway that triggers miR-23a upregulation in tumor tissue. Wang et al reported G6pc and $P g c-1 a$ as miR-23 direct targets demonstrating a complex IL-6/STAT3/miR-23/G6pc/Pgc-1 network responsible for reduced gluconeogenesis contributing to exacerbated cancer cells aerobic glycolysis in HCC. ${ }^{24}$

Abnormal lipid metabolism is a hallmark of cancer too, aiding cancer cell sustenance through accumulation of large amounts of FAs due to increased intracellular liponeogenesis or lipolysis. Liu et al reported an intriguing mechanism contributing to aberrant lipolysis in HCC by identifying the long non-coding RNA (lncRNA) NEAT1 
as a master regulator of adipose triglyceride lipase (ATGL) through miR-124-3p sequestration. ATGL initiates the metabolism of tri-acyl-glycerol (TAG) hydrolyzing it in diacyl-glycerol (DAG) and free FAs. It is upregulated in most of HCC, associates with poor survival and increases proliferation and tumorigenesis in preclinical models. ${ }^{25}$ These data emphasize the role of aberrant metabolism and activation of abnormal metabolic pathways as cofactors favoring malignant development. Unfortunately, this is still a poorly explored topic, which however holds promise as a source of biomarkers of increased risk of neoplastic development and prognosis linking metabolomics to miRNAomics profiles.

Rapid proliferation of tumor cells together with abnormal blood vessel generation is responsible for poor oxygen supply in the center of tumor masses leading to a hypoxic microenvironment. Cancer cell adaptation to hypoxic conditions requires gene modulation. Hypoxia influences miRNA biogenesis and expression ${ }^{26}$ and, in turn, miRNAs play a central role in the regulation of hypoxiaassociated pathways establishing entangled feedback loops that contribute to survival of cancer cells in hostile conditions. ${ }^{27}$ MiR-885-5p is downregulated in in vitro hypoxic models and its enforced expression decreases proliferation and migration capabilities and triggers apoptotic cell death. MiR-885-5p is a tumor suppressor miRNA downregulated in HCC. It influences the metabolic reprogramming of cancer cells through the direct regulation of hexokinase 2 (HK2), a critical enzyme that catalyzes the first irreversible and rate-limiting step of glycolysis, entrapping glucose inside the cells following its phosphorylation to glucose-6-phosphate (G6P). Both miR-885-5p and $\mathrm{HK} 2$ are independent risk factors for $\mathrm{HCC}$ and associated with a shorter overall survival, confirming the relevance of targeting metabolic pathways in $\mathrm{HCC}{ }^{28} \mathrm{HK} 2$ regulation by the hypoxia-induced miR-885-5p provides a link between hypoxia and metabolism in HCC cells. Remarkably, the standard of care in intermediate stage HCC is represented by TACE, which occludes the HCC feeding artery, causing acute hypoxia. We can hypothesize that the molecular events described previously might contribute to the partial or absent response to TACE in some cases, as well as to disease reactivation after an initial response. If confirmed, these findings might open the way to combined treatments with miRNA delivery inside the HCC nodule during TACE.

MiR-199a mature miRNAs are the third most expressed miRNAs in the liver, and among the most downregulated miRNAs in HCC tissue. These data provide the background for their therapeutic exploitation. ${ }^{29,30}$ MiR-199a-5p is a tumor suppressor miRNA able to bind to the 3 'UTR of HK2 mRNA contributing to regulate its expression and, consequently, to alter the glycometabolism. MiR-199a-5p overexpression determines lower levels of the glycolytic intermediate G6P and adenosine triphosphate (ATP). Due to the strict dependence of cancer cells from aerobic glycolysis, miR-199a-5p-mediated interference with this metabolic phenotype leads to impairment of cell proliferation in vitro and tumorigenic properties in vivo. Rescue experiments confirmed HK2 as being responsible for the anti-proliferative effect of miR-199a-5p and for mediating the miRNA-induced metabolic shift in HCC. Importantly, miR-199a-5p is associated with large tumor size, poor differentiation, high tumor-nodemetastasis (TNM) stage and tumor thrombus. Both low miR-199a-5p and high HK2 are independent risk factors in HCC. In the human genome, miR-199a is encoded by two loci, mir-199a-1 and mir-199a-2. Interestingly, hypoxia leads to decreased pri-mir-199a-1 transcript and mature miR-199a-5p levels through HIF-1 $\alpha$ binding to responsive elements (HRE) in pri-miR-199a-1 region, suggesting hypoxia as responsible for miRNA-mediated glycometabolic shift through HK2 targeting. ${ }^{31}$ By modulating two central glycolytic enzymes, HK2 and PKM2, miR-199a replacement therapy might exert a consistent anti-tumor effect, decreasing glucose uptake of cancer cells that can also be monitored by using PET/CT $\left(\left[{ }^{18} \mathrm{~F}\right]-\mathrm{FDG}\right)$ imaging. In this scenario, miR-199a-5p represents not only a promising candidate for a miRNA-based strategy but hypothetically may favor patient follow-up by imaging techniques. $^{32}$

Mutual regulation between miR-199a-5p and its target gene HIF- $1 \alpha$ has been reported in HCC cell lines establishing a positive feedback loop that leads to HIF-1 $\alpha$ downstream pathways' activation influencing metabolic reprogramming. In particular, under hypoxic conditions miR-199a-5p expression falls down to barely detectable levels in cardiomyocytes ${ }^{27}$ as well as in HCC cells where HIF- $1 \alpha$ activation per se is able to inhibit miRNA expression reinforcing glycolytic shift. Functional studies revealed a decrease of glucose uptake, lactate production and LDH activity in miR-199a-5p overexpressing HepG2 and Huh-7 cells together with a $\mathrm{pH}$ value increase in culture medium. HIF-1 $\alpha$ is a miR-199a-5p direct target in HCC orchestrating metabolic changes induced by miR-199a-5p itself. MiR-199a-5p not only decreases 
HIF- $1 \alpha$ protein expression but also impairs the transcriptional activation of several HIF-1 $\alpha$ downstream genes involved in glycolysis, such as glucose transporter 1 (GLUT1), HK2, PKM2 and lactate dehydrogenase A (LDHA). Interestingly miR-199a-5p exerts multi-target activity by regulating a single gene (HIF-1 $\alpha$ ) that, functioning as a transcription factor, amplifies miRNA downstream activity affecting the expression of multiple glucose-related genes. ${ }^{33}$ In addition, as previously reported, many HIF-1 $\alpha$ transcriptional genes are direct targets of miR-199a-5p itself (eg, HK2 and PKM2) reinforcing its final effect on aerobic glycolysis. MiR-199a-5p is an independent prognostic factor for HCC survival, displaying a strong inverse correlation with HIF-1 $\alpha$ in HCC specimens, confirming this feedback loop as pivotal in hepatocarcinogenesis. Strikingly, miR-199a-3p, the other mature miR-199a isoform, is also downregulated in HCC and triggers apoptotic cell death in hypoxic conditions through mTOR targeting, therefore concurring with the aggressiveness of HCC even in the absence of altered lactate production. ${ }^{31,34}$ Similarly, miR-139-5p is downregulated in human HCCs. Through the regulation of ETS1 transcription factor it mediates the regulation of the metabolic genes hexokinase 1 (HK1) and 6-phosphofructo-2-kinase/fructose-2,6-biphosphatase 3 (PFKFB3) controlling the metabolic shift of HCC cells by increasing glucose uptake and lactate production. ${ }^{35}$ Notably, miR$125 \mathrm{a}$ is another tumor suppressor miRNA downregulated under hypoxic conditions contributing to HK2 regulation, supporting the importance of hypoxia and HK2 in metabolic switch and HCC malignant phenotype. MiR-125a/ HK2 axis regulates glucose uptake as well as lactate, ATP and ROS production modulating cell growth and tumorigenic properties and contributing to the metabolic phenotype of HCC in hypoxic microenvironment. ${ }^{36} \mathrm{HIF}-1 \alpha$ stabilization is a central event during metabolic switch and molecules affecting this process might be considered as promising candidates for HCC treatment. The overexpression of tumor suppressor miR-592 inhibits aerobic glycolysis and cell proliferation by WD repeat and SOCS box containing 1 (WSB1) targeting, leading to HIF-1 $\alpha$ destabilization and impairing the Warburg effect of HCC cells. $^{37}$

The liver is an ideal organ to readily take-up nucleic acids, such as siRNAs and miRNAs. Therefore, the identification of miRNAs involved in tumor regression opens the path toward the development of small nucleic acidbased formulations as promising therapeutics. An intriguing example is represented by tumor suppressor miR-342-3p whose increased expression is a key event in $\mathrm{HCC}$ regression. The employment of conditional c-Myc transgenic (TG) models allowed gaining insight into miRNAs' involvement in tumor regression. MiR-342-3p resulted among the most upregulated ones in regressing lesions and its direct targeting of monocarboxylic acid transporter 1 (MCT1) influences cell metabolism and in particular lactate transport and glucose uptake. Indeed, miR-342-3p overexpression not only decreases proliferation and invasion capabilities but also reduces intracellular glucose and lactate in the presence of enhanced extracellular lactate levels, by interfering with MCT1 functions. TG mouse models demonstrated the long-term antitumor efficacy of adeno-associated vector (AAV8)-mediated miR-342-3p delivery system blocking tumor progression and driving increased survival, postulating miR-342-3p as a candidate for replacement therapy in $\mathrm{HCC} .{ }^{38}$ MiRNAs deeply affect the metabolic profile of HCC cells (Table 1) and appear to be promising targets for $\mathrm{HCC}$ treatment, adding a new dimension to the interference of cancer metabolism as a therapeutic option. Laboratory models are very useful to produce proof of concept studies. However, as detailed previously, the complex scenario in which multiple miRNAs converge and cooperate with hypoxia resistance and metabolic changes, reinforces the need to test miRNA panels instead of single miRNAs and to evaluate the modulation of more than one miRNA when replacement or inhibition strategies are to be considered.

\section{MicroRNAs and Microenvironment in HCC}

In the last decades, most studies focused on the effects of miRNAs in cancer development and progression, characterizing their intracellular functions and regulation of target genes. Recently, the activity of miRNAs in intercellular communication has been emerging as a crucial point in liver diseases promoting the recruitment of stromal and immune cells that play pivotal roles in tumor aggressiveness, metastasis and immune system evasion of cancer cells, providing a wealth of putative novel biomarkers. Indeed, tumor infiltrating cell populations can be actively recruited from the bone marrow, like endothelial progenitor cells, lymphocytes, myeloid cells and mesenchymal cells. Notably, once in the tumor microenvironment (TME), these infiltrating cells can be "educated" as in the case of tumor-associated macrophages 
Table I Metabolic-Associated miRNAs in HCC

\begin{tabular}{|l|c|c|c|}
\hline Deregulated miRNA & Target Genes & Metabolic Effect & Reference \\
\hline miR-I & G6PD & Increased Pentose Phosphate Pathway & Increased aerobic glycolysis \\
miR-122 & PKM2 & Increased aerobic glycolysis & {$[10]$} \\
miR-338 & PKLR, PKM2 & Decreased gluconeogenesis & {$[22,23]$} \\
miR-23 & G6PC; PgC-I & Increased lipolysis & {$[24]$} \\
miR-124-3p & ATGC & Increased aerobic glycolysis & {$[25]$} \\
miR-885-5p & HK2 & Increased aerobic glycolysis & {$[28]$} \\
miR-199a-5p & Increased aerobic glycolysis & {$[3 \mid-33]$} \\
miR-139-5p & ETSI & Increased aerobic glycolysis & {$[35]$} \\
miR-125a & HK2 & Increased aerobic glycolysis & {$[36]$} \\
miR-592 & WSBI & Impaired lactate transport & {$[37]$} \\
miR-342-3p & MCTI & {$[38]$} & \\
\hline
\end{tabular}

(TAMs) or cancer-associated fibroblasts (CAFs). This represents a dynamic and multi-lateral process that involves the participation of soluble molecules and microvesicles to promote active and bidirectional inter-cellular communication between cancer cells and the different TME cell populations that progressively switch toward their protumorigenic role (Figure 2). In this context, the development of therapeutic agents interfering with the recruitment of pro-tumorigenic cells or promoting the infiltration of immune-modulatory cells represents an interesting anticancer therapeutic option. In the following paragraphs we reported a few examples of the recruitment

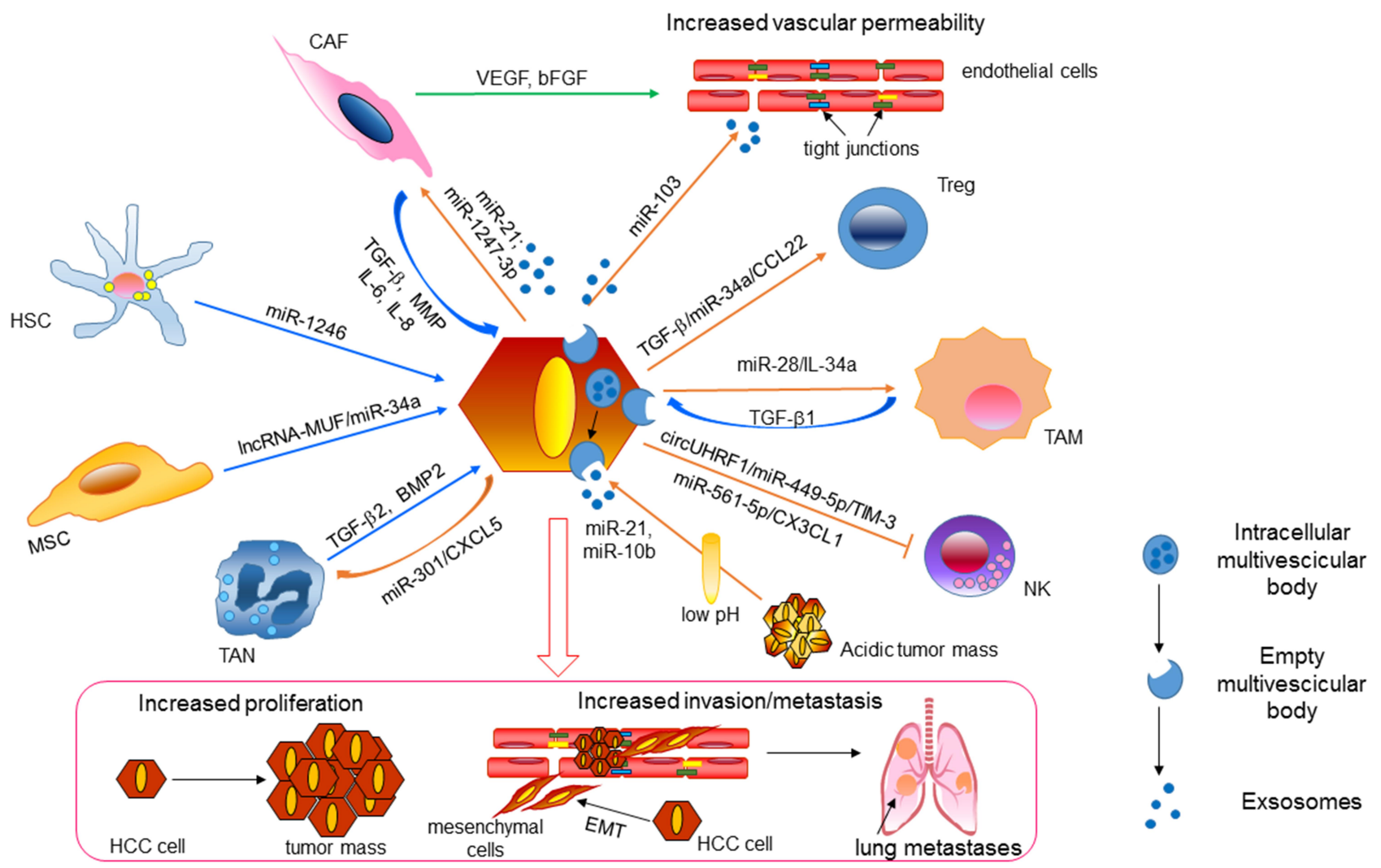

Figure 2 Bi-directional interactions between HCC cells and tumor microenvironment cell populations.

Notes: HCC cells secrete chemokines and cytokines to attract different cell types that acquire a pro-tumorigenic phenotype favoring HCC aggressiveness. MiRNAs and soluble factors take part in this complex intercellular crosstalk. Straight orange and blue arrows represent the initial signal from HCC or TME cells, respectively. Curved orange and blue arrows represent the feedback signal from HCC or TME cell, respectively.

Abbreviations: CAF, cancer-associated fibroblasts; Treg, T regulatory cells; TAM, tumor-associated macrophages; NK, natural killer lymphocytes; TAN, tumor-associated neutrophils; MSC, mesenchymal stem cells; HSC, hepatic stellate cells; EMT, epithelial-to-mesenchymal transition. 
of TME cells following the release of soluble factors by HCC cells as well as their feedback regulation of the cancer cell itself.

HBV and HCV infections are responsible for chronic inflammation and liver regeneration progressing from chronic hepatitis, fibrosis and cirrhosis to HCC. Intracellular miRNAs can guide the release of soluble chemokines/cytokines from cancer cells participating in a paracrine crosstalk between different cell types, thus contributing to leukocyte recruitment and tumor infiltration promoting tumor cell proliferation, metastasis and immune escape. TGF- $\beta$ signaling is activated in HBVassociated HCCs with portal vein tumor thrombosis and is involved in immune suppression through the recruitment of regulatory $\mathrm{T}$ (Treg) cells. In particular, TGF- $\beta$ is responsible for reduced miR-34a expression in HCC cells, which, in turn, was associated with increased risk of venous metastasis. ${ }^{39}$ Interestingly, miR-34a functional studies revealed no autonomous effect on cell proliferation, invasion and tumorigenic potential showing, on the other side, that miR-34a has impact on tumor microenvironment through the regulation of chemokine CCL22 that mediates Treg cells' recruitment into the tumor bulk promoting immune escape. ${ }^{40}$

MiR-28-5p is another metastasis-associated miRNA that exerts its paracrine activity by recruiting tumorinfiltrating macrophages through regulating interleukin 34 (IL-34) secretion. MiR-28-5p is downregulated in metastatic HCCs and its downregulation correlates with tumor metastasis, recurrence, and poor survival. MiR-28-5p deficiency results in the up-regulation of its direct target, IL34, which mediates tumor-associated macrophage (TAM) infiltration. On the other side, IL-34 further reduces miR$28-5 p$ expression following TGF- $\beta 1$ secretion by TAMs. The establishment of a miR-28-5p/IL-34/macrophage feedback loop potentiates the final effect of a prooncogenic signaling cascade that prevents miR-28-5p expression in HCC cells enhancing IL-34 secretion and macrophages' infiltration. ${ }^{41}$ Strikingly, modulating TAMs' switch from pro-tumorigenic M2-polarization toward an anti-tumor M1-like phenotype is emerging as a valid option for the treatment of cancers. Wang et al demonstrated that TAMs-targeted delivery of miR-99b in HCC and lung cancer models is sufficient to obtain TAMs' repolarization to M1-like immune suppressive phenotype by cellular reprogramming mediated by NF- $\mathrm{KB}$ activation and $\kappa \mathrm{B}-\mathrm{RAS} 2$ and mTOR direct targeting. ${ }^{42}$
Among tumor-infiltrating lymphocytes, natural killer (NK) cells are mediators of the innate immune response and contribute to the clearance of transformed cell clones. MiR-561-5p is a lung metastasis-associated miRNA that acts through a non-autonomous mechanism by targeting $\mathrm{CX}_{3} \mathrm{CL} 1$ chemokine in $\mathrm{HCC}$ cells. This chemokine triggers chemotactic migration and cytotoxicity of NK cells following STAT3 signaling activation. As proof of concept, the injection of $\mathrm{CX}_{3} \mathrm{CR} 1+\mathrm{NK}$ cells in a metastatic HCC mouse model reduced lung colonization and tumor foci. Notably, lung metastases displayed increased miR561-5p levels with respect to primary specimens, highlighting the contribution of miRNAs in modeling the cell composition of the metastatic niche avoiding immunesurveillance. ${ }^{43}$ In line with this, the exosomal secretion of circular ubiquitin-like with PHD and ring finger domain 1 RNA (circUHRF1) from cancer cells prevents NK tumor infiltration and blocks their anti-cancer function via miR$449 \mathrm{c}-5 \mathrm{p}$ sequestration and $\mathrm{T}$ cell immunoglobulin and mucin domain 3 (TIM-3) degradation, which has a critical role in immune evasion. CircUHRF1 expression is increased in HCC tissue, predicts poor prognosis, and is associated with decreased NK plasma levels. Exosomal circUhRF1 is internalized by NK cells determining their dysfunction due to decreased IFN- $\gamma$ and TNF- $\alpha$ secretion. As proof of principle, an increase in lung metastatic nodules was observed in circUHRF1 overexpressing PLC/PRF/5 xenograft model showing decreased NKG2positive infiltrating cells. Intriguingly, a negative correlation between circUHRF1 and NK cells was present in HCC patients with progressive disease, representing a promising target from a combined therapeutic perspective. $^{44}$

Intriguingly, an inverse crosstalk starting from TME cell populations and directed toward liver cancer cells can take place in liver tumors where the secretion of chemokines and cytokines, such as TGF- $\beta 2$ and bone morphogenetic protein 2 (BMP2) by tumor-associated neutrophils (TANs) drives miR-301b-3p expression in HCC recipient cells modifying their phenotypes. In more detail, miR301b-3p upregulation in TANs-activated HCC cells increases proliferation, migration, sphere formation, stemassociated genes and in vivo tumorigenicity through the direct regulation of CYLD lysine 63 deubiquitinase (CYLD) and limbic system-associated membrane protein (LSAMP). In addition, CYLD targeting activates NF- $\kappa \mathrm{B}$ signaling to promote chemokine CXCL5 release from HCC cells establishing a positive feedforward loop that 
further attracts TANs to infiltrate the tumor mass, contributing to tumor aggressiveness and poor prognosis. ${ }^{45}$ Cancer associated mesenchymal stem cells (MSCs) represent another tumor infiltrating cell population that, following co-culturing, influences cancer phenotype by increasing stemness and EMT properties of HCC cells through $\operatorname{lncRNA-MUF}$ (MSCs upregulated factor) upregulation in MSCs-HCC co-cultured cells. LncRNA-MUF performs its oncogenic function through both RNA and protein binding activity, triggering EMT process and tumor progression. Specifically, IncRNA-MUF binds to both Annexin A2 (ANXA2) and GSK-3 $\beta$ in the cytoplasm of HCC cells preventing the latter from $\beta$-catenin phosphorylation and degradation, leading to WNT signaling activation. On the other side, IncRNA-MUF exerts its competitive endogenous RNA (ceRNA) activity through sequestering miR-34a in $\mathrm{HCC}$ cells further promoting EMT program due to the upregulation of its target gene Snaill that influences tumor spreading through E-cadherin inhibition. ${ }^{46}$ Similarly, hepatic stellate cells (HSCs) modulate HCC malignant behavior by increasing onco-miR -1246 expression leading to retinoic acid receptor-related orphan receptor alpha $(\operatorname{ROR} \alpha)$ inhibition and $\mathrm{WNT} / \beta$ catenin signaling activation inducing epithelial-tomesenchymal conversion. In the liver, cancer-associated fibroblasts (CAFs) are mainly derived from HSCs playing a pivotal role in hepatocarcinogenesis due to cytokine and growth factor secretion that promotes HCC proliferation in a Treg-associated immune-suppressive context. ${ }^{47}$

Cancer cells can further modify the composition of the microenvironment milieu through the secretion of miRNAs embedded into nanoparticle-sized vesicles, termed extracellular vesicles (EV) and exosomes. The latter are small-sized (30-100 nm) EV released into the extracellular space upon fusion of multivesicular bodies to the plasma membrane. The peculiarity of EVs is their ability to carry cell-specific cargos of nucleic acids, proteins and lipids that can be selectively internalized by neighboring or distant recipient cells, reprogramming their activities. It is well known that tumor-secreted exosomes can influence angiogenesis, immune system infiltration, parenchymal remodeling to promote tumor proliferation and metastasis. Vice versa, extracellular stimuli and chemical composition of the tumor milieu might modulate the transcriptional profile of cancer cells as well as their secretion of cell-to-cell crosstalk molecules. Strikingly, an acidic extracellular microenvironment might influence exosome content and favor its release from cancer cells to stimulate HCC migration. Interestingly, the metabolic reprogramming of cancer cells (eg, aerobic glycolysis) is responsible for extracellular acidification creating a $\mathrm{pH}$ gradient from the inner tumor region to the surrounding normal tissue promoting metabolic adaptation through the activation of $\mathrm{pH}$ dependent proteins involved in tumor spreading to distant sites. Interestingly, acidic conditions increased intracellular and exosomal levels of miR-21 and miR-10b in an HIF- $1 \alpha /$ HIF- $2 \alpha$ transcriptional dependent manner. Isolated exosomes from HCC cells grown in acidic conditions showed in vitro and in vivo oncogenic properties triggering Vimentin and Snaill expression while downregulating PTEN and E-cadherin levels. Patients with early HCC (eHCC) showed increased miR-21 and miR$10 \mathrm{~b}$ exosomal levels with respect to healthy volunteers, associated with tumor size and recurrence. Multivariate analysis confirmed the prognostic potential of these two miRNAs in the survival of eHCC, whereas their targeting in vivo reduced tumor growth and lung metastasis, highlighting their role not only as prognostic markers but also as therapeutic targets. ${ }^{48}$ Regarding the influence of extracellular signals from HCC cells, exosomal miR-21 is a well-fitting example. MiR-21 is one of the most represented circulating miRNAs in HCC and its release from cancer cells impacts on different cell types of the extracellular milieu. Indeed, exosome-secreted miR-21 can reprogram HSC converting this major source of liver fibroblasts into CAFs, critical players that foster cancer progression through the secretion of pro-inflammatory and pro-angiogenic cytokines, such as VEGF, bFGF, TGF $\beta$, and metalloproteinases. ${ }^{49}$ Similarly, highly metastatic cancer cells secrete much more exosomes, which convert fibroblasts to CAFs improving their migratory potential and enhancing the expression of pro-inflammatory genes, such as IL-1 $\beta$, IL-8, IL-6 and different collagen types that, in turn, foster microenvironment inflammation and tumor progression. Exosomal-miR-1247-3p activates CAFs through the direct targeting of $\beta$-1,4-galactosyltransferases III (B4GALT3), a mediator of protein glycosylation whose deregulation promotes $\beta 1$-integrin stabilization and NF- $\kappa B$ signaling activation that leads to transcription of proinflammatory cytokines. IL-6 and IL- 8 secretion by CAFs take part in a bilateral interaction with cancer cells inducing enhanced stemness gene expression, spheroid formation, EMT, migration and sorafenib resistance. In human HCCs, exosomal miR-1247-3p is higher in lung metastatic patients with respect to non-metastatic ones 
whereas higher tissue levels correlate with AFP expression, cirrhosis, tumor thrombus and extra-hepatic metastasis. $^{50}$

Active angiogenesis and lack of endothelial cell integrity are two major factors further influencing tumor progression toward an advanced metastatic stage. Secreted miRNAs might also influence vascular integrity by weakening cell-to-cell adhesion contacts aiding tumor cells' extravasation and metastasis. High miR-103 serum levels are associated with higher metastatic potential, TNM stage, and recurrence risk and represent an independent predictor of shorter recurrence-free survival in $\mathrm{HCC}$ patients. HCC cells secrete exosome-associated miR-103 to be captured by endothelial cells where its overexpression directly blocks both adherence and tight junctionassociated molecules such as VE-Cad, p120, Zona Occludens 1 (ZO-1) increasing vascular permeability and, as a consequence, the number of circulating tumor cells and pulmonary metastatic foci in xenograft mice. In addition, miR-103 also plays an autonomous role in HCC cells where the loss of $\mathrm{p} 120 / \mathrm{E}$-cadherin/ $\beta$-catenin membrane-associated complex facilitates $\beta$-catenin nuclear translocation leading to WNT pathway activation and promoting cell migration. ${ }^{51}$

This network of events involving cancer drivers and miRNAs in the modulation of HCC cell metabolism, adaptation to hypoxia, modulation of the TME, displays clinical correlates that deserve attention. Understanding these mechanisms and defining their contribution in specific subgroups of patients might represent an additional tool both in biomarker research and in the identification of possible novel treatments. Due to the high complexity of miRNAs' interactions, miRNA panels instead of single miRNAs appear more promising as novel biomarkers or therapeutic targets.

\section{MicroRNAs and Immune-Oncology}

Immunotherapy targets immune-checkpoint molecules instead of cancer cells. The interaction between programmed cell death protein 1 (PD-1) and programmed death-ligand 1 (PD-L1) or programmed death-ligand 2 (PD-L2) inactivates $\mathrm{T}$ cells and halts the immune system reactions against cancer cells. Even though immunotherapy has opened a promising avenue for cancer treatment, the low response rate and the development of resistance still represent unmet needs. Knowing the mechanisms underneath immune-checkpoint regulation, might favor the understanding of resistance and help to develop biomarkers for patients' selection and response prediction from the perspective of possible combined strategies. Here we will focus on PD-1 and PD-L1 because they are the most commonly targeted immune checkpoints (ICPs) in HCC. MiRNAs can modulate PD-1 and PD-L1 expression either directly, by targeting the 3'UTR of their mRNAs, or indirectly, by modulating upstream pathways like PTEN/ PI3K/Akt or JAK/STAT (Figure 3). Post-transcriptional regulation by epigenetic changes strongly influences the overexpression of ICPs. ${ }^{52}$

In pancreatic cancer cells, miR-142-5p inhibits PDL1. ${ }^{53}$ Even though miR-142-5p targeting of PD-L1 has not been directly proven in HCC yet, this miRNA has been demonstrated to reduce cell viability and promote apoptosis in HCC-derived cell lines. ${ }^{54}$ In addition, its downregulation in HCC was associated with disease progression, while its restored expression suppressed HCC cell migration, emphasizing its role in HCC too. ${ }^{55}$ Thus, it is conceivable that miR-142-5p downregulation in HCC cells might contribute to increased expression of PD-L1, as reported for pancreatic cancer. Taken together, the findings of a downregulated expression of microRNA-142-5p in advanced HCC and its role in the modulation of PD-L1 expression warrant its validation in the setting of advanced HCC undergoing immunotherapy, aiming to identify possible combined treatments in specific patients' subgroups.

MiR-138 is another interesting miRNA, reported to target both PD-L1 and PD-1. In more detail, miR-138 directly inhibits PD-L1 in colorectal cancer, ${ }^{56}$ PD-L1 and PD-1 in NSCLC, ${ }^{57}$ and PD-1 in glioblastoma. ${ }^{58}$ Even though data on PD-L1 targeting have not been directly obtained in HCC, we know that this miRNA is downregulated in $\mathrm{HCC}$ and especially in $\mathrm{HBV}$-related cases, ${ }^{59}$ outlining this subgroup of HCC patients as the most suitable for possible miR-138 restoration. Remarkably, miR138 downregulation is associated with a worse prognosis and its restored expression drives anti-proliferative effects through targeting many oncogenic factors including cyclin D3, ${ }^{60}$ SOX9, ${ }^{61}$ SIRT $1{ }^{62}$ and others. Very recently, miR138 was found to target PD-1 in patients with HBV-related hepatitis, cirrhosis, and HCC. ${ }^{63}$ By restoring miR-138 expression in primary $\mathrm{T}$ cells, the authors obtained PD-1 inhibition and an increased cytokine secretion, suggesting that this approach might be promising not only in the setting of HBV infection, but also in the setting of HCC. Indeed, the simultaneous inhibition of PD-1 and PD-L1 and the accumulated data on the inhibition of other HCCdrivers make this miRNA very interesting for future 


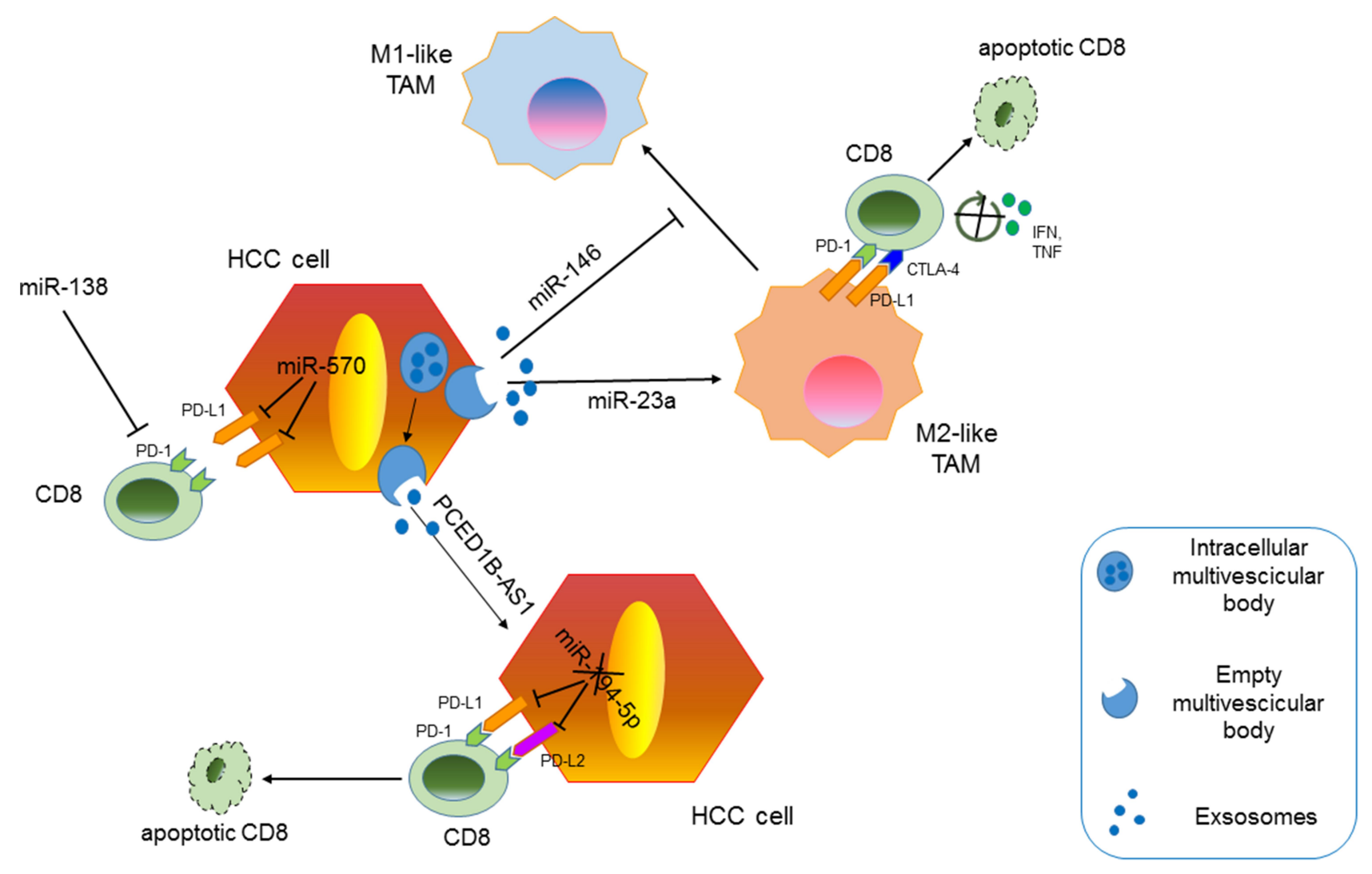

Figure 3 Immuno-modulatory function of HCC-specific miRNAs.

Notes: HCC cells release exosomes which RNA cargo (miRNAs, IncRNAs, mRNAS, etc.) is internalized by recipient cells modulating their phenotype and cell-cell interactions. TAM: tumor-associated macrophages with MI or M2-like phenotype; CD8: CD8+ T cells.

exploitations. Besides PD-1, miR-138 was also demonstrated to inhibit CTLA-4 by directly targeting its 3'UTRs in glioma cells, thus obtaining the simultaneous inhibition of another crucial ICP. ${ }^{58}$ Whether these findings can be extrapolated to HCC is still unknown and deserves further investigations.

Other miRNAs, such as miR-570, hold promise for future investigations as possible biomarkers or even therapeutic targets in patients with chronic liver diseases and HCC. MiR-570 emerged as the most relevantly deregulated miRNA in the progression of alcoholic liver disease to $\mathrm{HCC}$ by controlling the expression of 24 downstream genes, ${ }^{64}$ and it was demonstrated to inhibit HCC cell proliferation and invasion capability by targeting PDL1. ${ }^{65}$ In addition, Lin et al ${ }^{66}$ demonstrated miR-570's effects on apoptosis, angiogenesis, and $\mathrm{T}$ cell activation and proliferation in a mouse model obtained by HCCderived SMMC7721 cells injection. In this model, miR570 mimicked suppressed tumor growth, increased apoptotic-related factors, reduced CD31 and VEGF expression. Remarkably, a lower ratio of $\mathrm{CD}^{+} \mathrm{CD}^{+} \mathrm{T}$ cells and a higher ratio of $\mathrm{CD}^{+} \mathrm{IFN}_{-} \gamma^{+} \mathrm{T}$ cells were observed in peripheral blood and tumor tissues of miR-570 overexpressing tumors, suggesting a role in immune escape of HCC. Since PD-L1 and PD-L2 are both upregulated in $\mathrm{HCC}$ and share redundant functions, a common strategy targeting both of them holds promise from a therapeutic perspective. Fan et al reported the upregulation of lncRNAs PCED1B-AS1 in HCC tissue and demonstrated its sponging activity on miR-194-5p as functionally implicated in PD-L1/PD-L2 upregulation in HCC cells through an exosomal-mediated paracrine effect. Specifically, PCED1B-AS1 can be secreted in an exosomal-associated manner and picked-up by other recipient HCC cells where it exerts its oncogenic function by sequestering miR-194$5 \mathrm{p}$ that cannot further suppress PD-L1/PD-L2 expression, leading to their membrane localization. PD-L1/PD-L2 overexpression is therefore responsible for immune exhaustion of $\mathrm{T}$ cells due to increased apoptotic cell death and impaired IL-2 secretion. ${ }^{67}$ These findings need to be further validated in other HCC models as well as in the subgroup of human HCC characterized by immune- 
evasion profiles; however, they provide a preliminary background for future investigations on treatments aimed to control both the neoplastic changes and the immunerelated TME.

MiR-513, miR-34a, and miR-200 also display an inverse relationship with PD-L1 expression, even though they have been mostly investigated in other cancers rather than HCC. ${ }^{68-71}$ Notwithstanding, these miRNAs are deregulated in HCC where they play pivotal roles as drivers of neoplastic changes such as increased proliferation, reduced apoptosis, increased cell motility, EMT, and metabolic changes. ${ }^{72}$ In particular, miR-34 is downregulated in $\mathrm{HCC}^{73}$ where it modulates cell growth by inducing metabolic changes ${ }^{74}$ and influences the metastatic capability of HCC cells. ${ }^{75}$ Remarkably, miR-34 directly targets PD-L1 in acute myeloid leukemia ${ }^{70}$ and in NSCLC where its therapeutic delivery was shown to promote tumor-infiltrating lymphocytes (TILs), reduce $\mathrm{CD} 8(+)$ PD1 $(+)$ cells as well as radiation-induced macrophages and T-regulatory cells, supporting the possible combination of miRNAs with standard therapies. ${ }^{76}$ Collectively, these preliminary data suggest the rationale to investigate the expression of these miRNAs in different subsets of HCC, with particular regard to the immune-related and immune exhausted subgroups, in order to identify possible biomarkers of likelihood of response to ICPI, but also from the perspective of testing the effects of their restored expression in combined regimens.

As observed for PD-L1, PD-1 is modulated by miRNAs as well. Again, few data were directly generated in HCC patients; however, many of the PD-1-modulating miRNAs are well known players in HCC development and progression, thus representing a promising field of investigation. Besides the aforementioned targeting of PD-1 by miR-138 in HCC, we can recall the miR-15a/16 cluster, ${ }^{77,78} \mathrm{miR}-33 \mathrm{~b}$ and miR-28-5p. This last miRNA was described previously due to its ability to induce IL34 upregulation, contributing to tumor progression by TAM infiltration. ${ }^{41}$ In addition, miR-28-5p directly inhibited PD-1 in a melanoma mouse model, ${ }^{79}$ leading us to hypothesize similar mechanisms in HCC too. MiR-33b is downregulated in HCC tissues and its expression is lower in advanced stages. ${ }^{80}$ It is very interesting in the context of immune therapy due to its ability to directly target PD-1, at least in lung cancer cells. ${ }^{81}$ Besides PD-1 inhibition, miR$33 \mathrm{a}$ is also responsible for beta-catenin inhibition ${ }^{82}$ whose pathway activation is a frequent genetic lesion in HCC. If the immuno-modulatory function should also be confirmed in HCC, its restored expression might deserve attention in liver cancer as well as in other tumors, due to its ability to hit multiple driver pathways at one time.

HCC-derived exosome-embedded miRNAs might also take part in a regulatory crosstalk network favoring immune escape of cancer cells from CD8 + cells. The activation of specific molecules involved in the endoplasmic reticulum (ER) stress response often occurs in cancers contributing to foster tumor progression. MiR-23a is the most upregulated miRNA in exosomes from HCC cells treated with tunicamycin, an activator of the unfolded protein response (UPR), and it stimulates PD-L1 expression in tumor-infiltrating macrophages, leading to apoptotic cell death of T-cell population. Indeed, PTEN targeting by miR-23a activates Akt pathway which is responsible for PD-L1 expression in TAMs contributing to cancer cell immune evasion. ${ }^{83}$ Sal-like protein 4 (SALL4)-mediated transcriptional upregulation and exosomal internalization of miR-146a-5p is a further mechanism triggering macrophage remodeling toward an M2 phenotype due to NF-кB activation, switching from an anti-tumor immune response to pro-tumorigenic stimulation. In addition, exosomeeducated M2-polarized macrophages enhance the expression of PD-1 and CTLA-4 inhibitory receptors in CD3+ $\mathrm{T}$ cells leading to the impairment of IFN- $\gamma$ and TNF- $\alpha$ secretion. Since SALL4 is critical for miR-146a promoter regulation and exosomal enrichment, blocking SALL4/ miR-146a interaction represents a novel mechanism to prevent $\mathrm{T}$ cell exhaustion and delay HCC progression. ${ }^{84}$

In the context of the recent approval of ICP for the treatment of $\mathrm{HCC},{ }^{85}$ both intracellular and exosomalassociated miRNAs might represent promising targets restoring and potentiating the immune surveillance in the setting of novel anticancer strategies.

To sum up, even though very preliminary and needing further validation in HCC patients, the concomitant inhibition of HCC drivers and ICPs, further reinforce the interest toward exploitation of miRNAs as oncomarkers and possible therapeutic targets, in order to modulate both intracellular cancer-related pathways and immune tolerance promoters.

\section{Concluding Remarks}

In the early phases of miRNAs' research, their functions were mostly investigated in the context of simple inhibition of one or a few direct targets. We previously addressed the role of some HCC-specific miRNAs in cell cycle progression, promotion of invasion and angiogenesis as well as reduction of apoptotic cell death, contributing to 
hepatocarcinogenesis. We showed that aberrantly expressed miRNAs alter critical molecular pathways and biologic functions, suggesting their targeting as a novel strategy for HCC treatment. ${ }^{86}$ In the last years, a new look of miRNAs' contribution to the development of diseases has emerged. It is now accepted that miRNA final effects result from complex networks, often with feedback and feedforward loops, where the molecular background plays a pivotal role. In this regard, we previously summarized the interplay between deregulated miRNAs and TP53 highlighting the presence of mutual interactions. We also reported the dual behavior of some miRNAs (eg, miR-221 and miR-30e) in relationship with TP53 status emphasizing the need for an accurate genetic and molecular classification of HCC patients when a miRNA-based therapeutic option is taken into account. ${ }^{87}$ In particular, when considering a potential future translation of miRNAs into the clinic, we need to keep in mind the strict relationship between miRNAs' aberrant expression and the molecular background. Indeed, the concept of onco- and tumorsuppressor miRNAs should be contextualized as clarified by the so-called "dual miRNAs" which display divergent functions when acting in different contexts.

Many questions are still unanswered regarding the translational exploitation of miRNAs in HCC. Indeed, despite the great promise of preclinical studies, the translation of miRNA-based therapeutics into the clinic remains a tricky aspect affecting the success of clinical trials in oncologic patients. We previously described the representativeness of animal models with respect to human pathology and reported their employment in preclinical exploitations emphasizing their usefulness as tools for assessing the therapeutic potential of miRNA-mediated strategies in HCC. ${ }^{88}$ In this scenario, the field that appears to hold more promise in the short run is that of miRNAs as novel biomarkers, while their therapeutic exploitation is still a work in progress. Indeed, miRNAs' manipulation in clinical trials has shown the effectiveness of target modulation as well as some effects on disease progression. However, delivery still represents a challenge for miRNAs therapeutics, as observed in the case of MRX34 trial (NCT01829971 - Phase 1 study of MRX34), a liposomal miR-34a mimic, in patients with advanced solid tumors which was stopped due to serious immunemediated adverse events. ${ }^{89}$ Remarkably, different formulations such as those used to deliver miR-122 (NCT01200420), showed that a hepatocyte targeted $\mathrm{N}$-acetylgalactosamine conjugated anti-miR-122 oligonucleotide is effective in viral load reductions and is well tolerated when administered to patients with hepatitis $\mathrm{C}$ virus infection..$^{90}$ Encouraging findings were reported from the MesoMiR-1, a phase 1 trial (NCT02369198) delivering miRNA mimics in thoracic cancer patients based on the miR-15/107 consensus sequence. ${ }^{91}$ MiR-15/ 107 consensus sequence were packaged in a bacterially derived delivery system $^{92}$ named EDV-TM nanocells (EDVs), targeted with an anti-EGFR antibody. This study proved the acceptable tolerability and safety profile of this administration route. An issue to keep in mind when dealing with $\mathrm{HCC}$ is the high avidity of liver cancer cells toward the uptake of small RNAs. In addition, HCC nodules can be reached easily and selectively for drug administration by injection in the tumor-feeding artery as it happens in the current standard care of patients. Thus, HCC might be favored over other cancers from this perspective.

Notwithstanding, much evidence still needs to be validated in HCC and specifically, in defined subgroups of HCC, before considering a possible "bench-to-bedside" translation. The promising success of such kind of treatments mostly relies on an accurate selection of molecular backgrounds suitable for gene expression modulation. Here, we have addressed the importance of molecular contexts in miRNA biologic functions and we have described miRNA involvement in metabolic rewiring and crosstalk between HCC cells and TME or immune system cells as key aspects for the identification of novel therapeutic options. Remarkably, recent advances highlight the strict relationships and convergence of multiple miRNAs in the modulation of common pathways, signaling cascades, and phenotypic changes. This clearly indicates that miRNA panels over single miRNAs should be considered both from the perspective of biomarker identification and therapeutic exploitation.

\section{Acknowledgments}

This work was supported by funds from the Italian Association for Cancer Research (AIRC IG-25187, "Identification of circulating biomarkers for patient allocation to the best treatment in hepatocellular carcinoma") to FF and Programma di Ricerca Regione-Università, Regione Emilia Romagna, Bando "Ricerca Innovativa" (Innovative approaches to the diagnosis and pharmacogenetics-based therapies of primary hepatic tumors, peripheral $\mathrm{B}$ and T-cell lymphomas and lymphoblastic leukaemias) to LG. 


\section{Disclosure}

Prof. Dr. Fabio Piscaglia report Esaote (Genova, Italia): Research contract with University; Personal fees from AstraZeneca, Roche, Alkermes: advisory board. Bayer, Bracco, EISAI, IPSEN, MSD, BMS: speaker bureau, advisory board. GE, Tiziana Life Sciences: consultant. Samsung: speaker bureau. The Authors report no other conflicts of interest in this work.

\section{References}

1. Petrick JL, McGlynn KA. The changing epidemiology of primary liver cancer. Curr Epidemiol Rep. 2019;6(2):104-111. doi:10.1007/ s40471-019-00188-3

2. Toffanin S, Hoshida Y, Lachenmayer A, et al. MicroRNA-based classification of hepatocellular carcinoma and oncogenic role of miR-517a. Gastroenterology. 2011;140(5):1618-28 e16. doi:10.1053/j.gastro.2011.02.009

3. Morishita A, Oura K, Tadokoro T, Fujita K, Tani J, Masaki T. MicroRNAs in the pathogenesis of hepatocellular carcinoma: a review. Cancers. 2021;13(3):514. doi:10.3390/cancers13030514

4. Fornari F, Milazzo M, Chieco P, et al. In hepatocellular carcinoma miR-519d is up-regulated by p53 and DNA hypomethylation and targets CDKN1A/p21, PTEN, AKT3 and TIMP2. J Pathol. 2012;227(3):275-285. doi:10.1002/path.3995

5. Flor I, Bullerdiek J. The dark side of a success story: microRNAs of the C19MC cluster in human tumours. J Pathol. 2012;227 (3):270-274. doi:10.1002/path.4014

6. Gramantieri L, Pollutri D, Gagliardi M, et al. MiR-30e-3p influences tumor phenotype through MDM2/TP53 axis and predicts sorafenib resistance in hepatocellular carcinoma. Cancer Res. 2020;80 (8):1720-1734. doi:10.1158/0008-5472.CAN-19-0472

7. Hanahan D, Weinberg RA. Hallmarks of cancer: the next generation. Cell. 2011;144(5):646-674. doi:10.1016/j.cell.2011.02.013

8. Warburg O. On respiratory impairment in cancer cells. Science. 1956;124(3215):269-270.

9. Denko NC. Hypoxia, HIF1 and glucose metabolism in the solid tumour. Nat Rev Cancer. 2008;8(9):705-713. doi:10.1038/nrc2468

10. Kowalik MA, Guzzo G, Morandi A, et al. Metabolic reprogramming identifies the most aggressive lesions at early phases of hepatic carcinogenesis. Oncotarget. 2016;7(22):32375-32393. doi:10.18632/ oncotarget.8632

11. Chen Z, Lu W, Garcia-Prieto C, Huang P. The Warburg effect and its cancer therapeutic implications. J Bioenerg Biomembr. 2007;39 (3):267-274. doi:10.1007/s10863-007-9086-x

12. Poff A, Koutnik AP, Egan KM, Sahebjam S, D'Agostino D, Kumar NB. Targeting the Warburg effect for cancer treatment: ketogenic diets for management of glioma. Semin Cancer Biol. 2019;56:135-148. doi:10.1016/j.semcancer.2017.12.011

13. Schwartz L, Seyfried T, Alfarouk KO, Da Veiga Moreira J, Fais S. Out of Warburg effect: an effective cancer treatment targeting the tumor specific metabolism and dysregulated pH. Semin Cancer Biol. 2017;43:134-138. doi:10.1016/j.semcancer.2017.01.005

14. Wang ZEK, Yang Q, Qin L, et al. A novel metabolism-related signature as a candidate prognostic biomarker for hepatocellular carcinoma. $J$ Hepatocell Carcinoma. 2021;2021(8):119-132. doi:10.2147/JHC.S294108

15. Lai CY, Yeh KY, Lin CY, et al. MicroRNA-21 plays multiple oncometabolic roles in the process of NAFLD-related hepatocellular carcinoma via PI3K/AKT, TGF-beta, and STAT3 signaling. Cancers. 2021;13(5):940. doi:10.3390/cancers13050940
16. Esau C, Davis S, Murray SF, et al. miR-122 regulation of lipid metabolism revealed by in vivo antisense targeting. Cell Metab. 2006;3(2):87-98. doi:10.1016/j.cmet.2006.01.005

17. Tsai WC, Hsu SD, Hsu CS, et al. MicroRNA-122 plays a critical role in liver homeostasis and hepatocarcinogenesis. J Clin Investig. 2012;122(8):2884-2897. doi:10.1172/JCI63455

18. Chai C, Rivkin M, Berkovits L, et al. Metabolic circuit involving free fatty acids, microRNA 122, and triglyceride synthesis in liver and muscle tissues. Gastroenterology. 2017;153(5):1404-1415. doi:10.1053/j.gastro.2017.08.013

19. Janssen HL, Reesink HW, Lawitz EJ, et al. Treatment of HCV infection by targeting microRNA. New Engl J Med. 2013;368 (18):1685-1694. doi:10.1056/NEJMoa1209026

20. Burchard J, Zhang C, Liu AM, et al. microRNA-122 as a regulator of mitochondrial metabolic gene network in hepatocellular carcinoma. Mol Syst Biol. 2010;6(1):402. doi:10.1038/msb.2010.58

21. Liu AM, Xu Z, Shek FH, et al. miR-122 targets pyruvate kinase M2 and affects metabolism of hepatocellular carcinoma. PLoS One. 2014;9(1):e86872. doi:10.1371/journal.pone.0086872

22. Nie H, Li J, Yang XM, et al. Mineralocorticoid receptor suppresses cancer progression and the Warburg effect by modulating the miR-338-3p-PKLR axis in hepatocellular carcinoma. Hepatology. 2015;62(4):1145-1159. doi:10.1002/hep.27940

23. Li Q, Pan X, Zhu D, Deng Z, Jiang R, Wang X. Circular RNA MAT2B promotes glycolysis and malignancy of hepatocellular carcinoma through the miR-338-3p/PKM2 axis under hypoxic stress. Hepatology. 2019;70(4):1298-1316. doi:10.1002/hep.30671

24. Wang B, Hsu SH, Frankel W, Ghoshal K, Jacob ST. Stat3-mediated activation of microRNA-23a suppresses gluconeogenesis in hepatocellular carcinoma by down-regulating glucose-6-phosphatase and peroxisome proliferator-activated receptor gamma, coactivator 1 alpha. Hepatology. 2012;56(1):186-197. doi:10.1002/hep.25632

25. Liu X, Liang $Y$, Song $R$, et al. Long non-coding RNA NEAT1-modulated abnormal lipolysis via ATGL drives hepatocellular carcinoma proliferation. Mol Cancer. 2018;17(1):90. doi:10.1186/ s12943-018-0838-5

26. Nallamshetty S, Chan SY, Loscalzo J. Hypoxia: a master regulator of microRNA biogenesis and activity. Free Radic Biol Med. 2013;64:20-30. doi:10.1016/j.freeradbiomed.2013.05.022

27. Rane S, He M, Sayed D, et al. Downregulation of miR-199a derepresses hypoxia-inducible factor-1alpha and Sirtuin 1 and recapitulates hypoxia preconditioning in cardiac myocytes. Circ Res. 2009;104(7):879-886. doi:10.1161/CIRCRESAHA.108.193102

28. Xu F, Yan JJ, Gan Y, et al. miR-885-5p negatively regulates warburg effect by silencing hexokinase 2 in liver cancer. Mol Ther Nucleic Acids. 2019;18:308-319. doi:10.1016/j.omtn.2019.09.002

29. Callegari E, D'Abundo L, Guerriero P, et al. miR-199a-3p modulates MTOR and PAK4 pathways and inhibits tumor growth in a hepatocellular carcinoma transgenic mouse model. Mol Ther Nucleic Acids. 2018;11:485-493. doi:10.1016/j.omtn.2018.04.002

30. Hou J, Lin L, Zhou W, et al. Identification of miRNomes in human liver and hepatocellular carcinoma reveals miR-199a/b-3p as therapeutic target for hepatocellular carcinoma. Cancer Cell. 2011;19 (2):232-243. doi:10.1016/j.ccr.2011.01.001

31. Guo W, Qiu Z, Wang Z, et al. MiR-199a-5p is negatively associated with malignancies and regulates glycolysis and lactate production by targeting hexokinase 2 in liver cancer. Hepatology. 2015;62 (4):1132-1144. doi:10.1002/hep.27929

32. Zhang LF, Lou JT, Lu MH, et al. Suppression of miR-199a maturation by HuR is crucial for hypoxia-induced glycolytic switch in hepatocellular carcinoma. EMBO J. 2015;34(21):2671-2685. doi:10.15252/embj.201591803

33. Li B, He L, Zuo D, et al. Mutual regulation of MiR-199a-5p and HIF-1alpha modulates the warburg effect in hepatocellular carcinoma. J Cancer. 2017;8(6):940-949. doi:10.7150/jca.17496 
34. Fornari F, Milazzo M, Chieco P, et al. MiR-199a-3p regulates mTOR and c-Met to influence the doxorubicin sensitivity of human hepatocarcinoma cells. Cancer Res. 2010;70(12):5184-5193. doi:10.1158/ 0008-5472.CAN-10-0145

35. Hua S, Lei L, Deng L, et al. miR-139-5p inhibits aerobic glycolysis, cell proliferation, migration, and invasion in hepatocellular carcinoma via a reciprocal regulatory interaction with ETS1. Oncogene. 2018;37(12):1624-1636. doi:10.1038/s41388-017-0057-3

36. Jin F, Wang Y, Zhu Y, et al. The miR-125a/HK2 axis regulates cancer cell energy metabolism reprogramming in hepatocellular carcinoma. Sci Rep. 2017;7(1):3089. doi:10.1038/s41598-017-03407-3

37. Jia YY, Zhao JY, Li BL, et al. miR-592/WSB1/HIF-1alpha axis inhibits glycolytic metabolism to decrease hepatocellular carcinoma growth. Oncotarget. 2016;7(23):35257-35269. doi:10.18632/ oncotarget.9135

38. Komoll RM, Hu Q, Olarewaju O, et al. MicroRNA-342-3p is a potent tumour suppressor in hepatocellular carcinoma. J Hepatol. 2021;74 (1):122-134. doi:10.1016/j.jhep.2020.07.039

39. Budhu A, Jia HL, Forgues $M$, et al. Identification of metastasis-related microRNAs in hepatocellular carcinoma. Hepatology. 2008;47(3):897-907. doi:10.1002/hep.22160

40. Yang P, Li QJ, Feng Y, et al. TGF-beta-miR-34a-CCL22 signaling-induced Treg cell recruitment promotes venous metastases of HBV-positive hepatocellular carcinoma. Cancer Cell. 2012;22 (3):291-303. doi:10.1016/j.ccr.2012.07.023

41. Zhou SL, Hu ZQ, Zhou ZJ, et al. miR-28-5p-IL-34-macrophage feedback loop modulates hepatocellular carcinoma metastasis. Hepatology. 2016;63(5):1560-1575. doi:10.1002/hep.28445

42. Wang L, Hu YY, Zhao JL, et al. Targeted delivery of miR-99b reprograms tumor-associated macrophage phenotype leading to tumor regression. J Immunother Cancer. 2020;8(2):e000517. doi:10.1136/jitc-2019-000517

43. Chen EB, Zhou ZJ, Xiao K, et al. The miR-561-5p/CX3CL1 signaling axis regulates pulmonary metastasis in hepatocellular carcinoma involving CX3CR1(+) natural killer cells infiltration. Theranostics. 2019;9(16):4779-4794. doi:10.7150/thno.32543

44. Zhang PF, Gao C, Huang XY, et al. Cancer cell-derived exosomal circUHRF1 induces natural killer cell exhaustion and may cause resistance to anti-PD1 therapy in hepatocellular carcinoma. Mol Cancer. 2020;19(1):110. doi:10.1186/s12943-020-01222-5

45. Zhou SL, Yin D, Hu ZQ, et al. A positive feedback loop between cancer stem-like cells and tumor-associated neutrophils controls hepatocellular carcinoma progression. Hepatology. 2019;70 (4):1214-1230. doi:10.1002/hep.30630

46. Yan $\mathrm{X}$, Zhang D, Wu W, et al. Mesenchymal stem cells promote hepatocarcinogenesis via IncRNA-MUF interaction with ANXA2 and miR-34a. Cancer Res. 2017;77(23):6704-6716. doi:10.1158/00085472.CAN-17-1915

47. Huang JL, Fu YP, Gan W, et al. Hepatic stellate cells promote the progression of hepatocellular carcinoma through microRNA-1246RORalpha-Wnt/beta-Catenin axis. Cancer Lett. 2020;476:140-151. doi:10.1016/j.canlet.2020.02.012

48. Tian XP, Wang CY, Jin XH, et al. Acidic microenvironment up-regulates exosomal miR-21 and miR-10b in early-stage hepatocellular carcinoma to promote cancer cell proliferation and metastasis. Theranostics. 2019;9(7):1965-1979. doi:10.7150/ thno.30958

49. Zhou Y, Ren H, Dai B, et al. Hepatocellular carcinoma-derived exosomal miRNA-21 contributes to tumor progression by converting hepatocyte stellate cells to cancer-associated fibroblasts. J Exp Clin Cancer Res. 2018;37(1):324. doi:10.1186/s13046-018-0965-2

50. Fang T, Lv H, Lv G, et al. Tumor-derived exosomal miR-1247-3p induces cancer-associated fibroblast activation to foster lung metastasis of liver cancer. Nat Commun. 2018;9(1):191. doi:10.1038/ s41467-017-02583-0
51. Fang JH, Zhang ZJ, Shang LR, et al. Hepatoma cell-secreted exosomal microRNA-103 increases vascular permeability and promotes metastasis by targeting junction proteins. Hepatology. 2018;68 (4):1459-1475. doi:10.1002/hep.29920

52. Omar HA, El-Serafi AT, Hersi F, et al. Immunomodulatory MicroRNAs in cancer: targeting immune checkpoints and the tumor microenvironment. FEBS J. 2019;286(18):3540-3557. doi:10.1111/ febs. 15000

53. Jia L, Xi Q, Wang H, et al. miR-142-5p regulates tumor cell PD-L1 expression and enhances anti-tumor immunity. Biochem Biophys Res Commun. 2017;488(2):425-431. doi:10.1016/j.bbrc.2017.05.074

54. Lou K, Chen N, Li Z, et al. MicroRNA-142-5p overexpression inhibits cell growth and induces apoptosis by regulating FOXO in hepatocellular carcinoma cells. Oncol Res. 2017;25(1):65-73. doi:10.3727/096504016X14719078133366

55. Tsang FH, Au SL, Wei L, et al. MicroRNA-142-3p and microRNA-142-5p are downregulated in hepatocellular carcinoma and exhibit synergistic effects on cell motility. Front Med. 2015;9 (3):331-343. doi:10.1007/s11684-015-0409-8

56. Zhao L, Yu H, Yi S, et al. The tumor suppressor miR-138-5p targets PD-L1 in colorectal cancer. Oncotarget. 2016;7(29):45370-45384. doi:10.18632/oncotarget.9659

57. Song N, Li P, Song P, et al. MicroRNA-138-5p suppresses non-small cell lung cancer cells by targeting PD-L1/PD-1 to regulate tumor microenvironment. Front Cell Dev Biol. 2020;8:540. doi:10.3389/ fcell. 2020.00540

58. Wei J, Nduom EK, Kong LY, et al. MiR-138 exerts anti-glioma efficacy by targeting immune checkpoints. Neuro Oncol. 2016;18 (5):639-648. doi:10.1093/neuonc/nov292

59. Li J, Shi W, Gao Y, et al. Analysis of microRNA expression profiles in human hepatitis B virus-related hepatocellular carcinoma. Clin Lab. 2013;59(9-10):1009-1015. doi:10.7754/Clin.Lab.2012.120901

60. Huang B, Li H, Huang L, Luo C, Zhang Y. Clinical significance of microRNA 138 and cyclin D3 in hepatocellular carcinoma. J Surg Res. 2015;193(2):718-723. doi:10.1016/j.jss.2014.03.076

61. Liu Y, Zhang W, Liu K, Liu S, Ji B, Wang Y. miR-138 suppresses cell proliferation and invasion by inhibiting SOX9 in hepatocellular carcinoma. Am J Transl Res. 2016;8(5):2159-2168.

62. Luo J, Chen P, Xie W, Wu F. MicroRNA-138 inhibits cell proliferation in hepatocellular carcinoma by targeting Sirt1. Oncol Rep. 2017;38(2):1067-1074. doi:10.3892/or.2017.5782

63. Liu W, Zheng X, Wang J, et al. MicroRNA-138 regulates T-cell function by targeting PD-1 in patients with hepatitis B virus-related liver diseases. Lab Med. 2021;epub ahed of print. doi:10.1093/ labmed/lmaa110

64. Liu Y, Chen SH, Jin X, Li YM. Analysis of differentially expressed genes and microRNAs in alcoholic liver disease. Int $\mathrm{J} \mathrm{Mol} \mathrm{Med.}$ 2013;31(3):547-554. doi:10.3892/ijmm.2013.1243

65. Guo W, Tan W, Liu S, et al. MiR-570 inhibited the cell proliferation and invasion through directly targeting B7-H1 in hepatocellular carcinoma. Tumour Biol. 2015;36(11):9049-9057. doi:10.1007/ s13277-015-3644-3

66. Lin Y, Liu S, Su L, et al. miR-570 inhibits proliferation, angiogenesis, and immune escape of hepatocellular carcinoma. Cancer Biother Radiopharm. 2018;33(6):252-257. doi:10.1089/cbr.2017.2389

67. Fan F, Chen K, Lu X, Li A, Liu C, Wu B. Dual targeting of PD-L1 and PD-L2 by PCED1B-AS1 via sponging hsa-miR-194-5p induces immunosuppression in hepatocellular carcinoma. Hepatol Int. 2020; online ahed of print.

68. Chen L, Gibbons DL, Goswami S, et al. Metastasis is regulated via microRNA-200/ZEB1 axis control of tumour cell PD-L1 expression and intratumoral immunosuppression. Nat Commun. 2014;5(1):5241. doi: $10.1038 /$ ncomms 6241

69. Chen XM. MicroRNA signatures in liver diseases. World $J$ Gastroenterol. 2009;15(14):1665-1672. doi:10.3748/wjg.15.1665 
70. Wang X, Li J, Dong K, et al. Tumor suppressor miR-34a targets PD-L1 and functions as a potential immunotherapeutic target in acute myeloid leukemia. Cell Signal. 2015;27(3):443-452. doi:10.1016/j. cellsig.2014.12.003

71. Wrangle J, Wang W, Koch A, et al. Alterations of immune response of non-small cell lung cancer with azacytidine. Oncotarget. 2013;4 (11):2067-2079. doi:10.18632/oncotarget.1542

72. Mao Y, Chen W, Wu H, Liu C, Zhang J, Chen S. Mechanisms and functions of MiR-200 family in hepatocellular carcinoma. Onco Targets Ther. 2020;13:13479-13490. doi:10.2147/OTT.S288791

73. Xie K, Liu J, Chen J, et al. Methylation-associated silencing of microRNA-34b in hepatocellular carcinoma cancer. Gene. 2014;543 (1):101-107. doi:10.1016/j.gene.2014.03.059

74. Zhang HF, Wang YC, Han YD. MicroRNA34a inhibits liver cancer cell growth by reprogramming glucose metabolism. Mol Med Rep. 2018;17(3):4483-4489. doi:10.3892/mmr.2018.8399

75. Wei W, Tang H, Tang L. MicroRNA-34a inhibits metastasis in liver cancer cells. Oncol Lett. 2018;16(6):6960-6965. doi:10.3892/ ol.2018.9555

76. Cortez MA, Ivan C, Valdecanas D, et al. PDL1 regulation by p53 via miR-34. J Natl Cancer Inst. 2016;108(1).

77. Cheng B, Ding F, Huang CY, Xiao H, Fei FY, Li J. Role of miR-16$5 \mathrm{p}$ in the proliferation and metastasis of hepatocellular carcinoma. Eur Rev Med Pharmacol Sci. 2019;23(1):137-145. doi:10.26355/ eurrev_201901_16757

78. Yang J, Liu R, Deng Y, et al. MiR-15a/16 deficiency enhances anti-tumor immunity of glioma-infiltrating CD8+ T cells through targeting mTOR. Int $J$ Cancer. 2017;141(10):2082-2092. doi:10.1002/ijc.30912

79. Li Q, Johnston $\mathrm{N}$, Zheng $\mathrm{X}$, et al. miR-28 modulates exhaustive differentiation of $\mathrm{T}$ cells through silencing programmed cell death-1 and regulating cytokine secretion. Oncotarget. 2016;7 (33):53735-53750. doi:10.18632/oncotarget.10731

80. Tian Q, Xiao Y, Wu Y, et al. MicroRNA-33b suppresses the proliferation and metastasis of hepatocellular carcinoma cells through the inhibition of Sal-like protein 4 expression. Int J Mol Med. 2016;38 (5):1587-1595. doi:10.3892/ijmm.2016.2754

81. Boldrini L, Giordano M, Niccoli C, et al. Role of microRNA-33a in regulating the expression of $\mathrm{PD}-1$ in lung adenocarcinoma. Cancer Cell Int. 2017;17(1):105. doi:10.1186/s12935-017-0474-y
82. Zhu C, Zhao Y, Zhang Z, Ni Y, Li X, Yong H. MicroRNA-33a inhibits lung cancer cell proliferation and invasion by regulating the expression of beta-catenin. Mol Med Rep. 2015;11(5):3647-3651. doi:10.3892/mmr.2014.3134

83. Liu J, Fan L, Yu H, et al. Endoplasmic reticulum stress causes liver cancer cells to release exosomal miR-23a-3p and up-regulate programmed death ligand 1 expression in macrophages. Hepatology. 2019;70(1):241-258. doi:10.1002/hep.30607

84. Yin C, Han Q, Xu D, Zheng B, Zhao X, Zhang J. SALL4-mediated upregulation of exosomal miR-146a-5p drives T-cell exhaustion by M2 tumor-associated macrophages in HCC. Oncoimmunol. 2019;8 (7):1601479. doi:10.1080/2162402X.2019.1601479

85. Finn RS, Qin S, Ikeda M, et al. Atezolizumab plus bevacizumab in unresectable hepatocellular carcinoma. New Engl J Med. 2020;382 (20):1894-1905. doi:10.1056/NEJMoa1915745

86. Gramantieri L, Fornari F, Callegari E, et al. MicroRNA involvement in hepatocellular carcinoma. J Cell Mol Med. 2008;12 (6A):2189-2204. doi:10.1111/j.1582-4934.2008.00533.x

87. Pollutri D, Gramantieri L, Bolondi L, Fornari F. TP53/MicroRNA interplay in hepatocellular carcinoma. Int J Mol Sci. 2016;17:12. doi:10.3390/ijms 17122029

88. Fornari F, Gramantieri L, Callegari E, et al. MicroRNAs in animal models of HCC. Cancers. 2019;11(12):1906. doi:10.3390/ cancers 11121906

89. Hong DS, Kang YK, Borad M, et al. Phase 1 study of MRX34, a liposomal miR-34a mimic, in patients with advanced solid tumours. Br J Cancer. 2020;122(11):1630-1637. doi:10.1038/s41416-0200802-1

90. van der Ree MH, de Vree JM, Stelma F, et al. Safety, tolerability, and antiviral effect of RG-101 in patients with chronic hepatitis $\mathrm{C}$ : a phase 1B, double-blind, randomised controlled trial. Lancet. 2017;389(10070):709-717. doi:10.1016/S0140-6736(16)31715-9

91. Reid G, Kao SC, Pavlakis N, et al. Clinical development of TargomiRs, a miRNA mimic-based treatment for patients with recurrent thoracic cancer. Epigenomics. 2016;8(8):1079-1085. doi:10.2217/epi-2016-0035

92. MacDiarmid JA, Mugridge NB, Weiss JC, et al. Bacterially derived $400 \mathrm{~nm}$ particles for encapsulation and cancer cell targeting of chemotherapeutics. Cancer Cell. 2007;11(5):431-445. doi:10.1016/j. ccr.2007.03.012
Journal of Hepatocellular Carcinoma

\section{Publish your work in this journal}

The Journal of Hepatocellular Carcinoma is an international, peerreviewed, open access journal that offers a platform for the dissemination and study of clinical, translational and basic research findings in this rapidly developing field. Development in areas including, but not limited to, epidemiology, vaccination, hepatitis therapy, pathology and molecular tumor classification and prognostication are all considered for publication. The manuscript management system is completely online and includes a very quick and fair peer-review system, which is all easy to use. Visit http://www.dovepress.com/ testimonials.php to read real quotes from published authors. 